\title{
Development of Simple Articulated Human Models using Superquadrics for Dynamic Analysis
}

\author{
Hyun Min Lee ${ }^{1}$, Jay Jung Kim ${ }^{1}$, Je Wook Chae ${ }^{2}$ \\ ${ }^{1}$ School of Mechanical Engineering, Hanyang University, Seoul, 133-791 \\ ${ }^{2}$ Agency for Defense Development, Daejeon, 305-152
}

\begin{abstract}
Objective: This study is aimed at developing Articulated Human Models(AHM) using superquadrics to improve the geometric accuracy of the body shape. Background: The previous work presents the AHM with geometrical simplification such as ellipsoids to improve analysis efficiency. However, because of the simplicity, their physical properties such as a center of mass and moment of inertia are computed with errors compared to their actual values. Method: This paper introduces a three steps method to present the AHM with superquadrics. First, a 3D whole body scan data are divided into 17 body segments according to body joints. Second, superquadric fitting is employed to minimize the Euclidean distance between body segments and superquadrics. Finally, Fee-Form Deformation is used to improve accuracy over superquadric fitting. Results: Our computational experiment shows that the superquadric models give better accuracy of dynamic analysis than that of ellipsoid ones. Conclusion: We generate the AHM composed of 17 superquadrics and 16 joints using superquadric fitting. Application: The AHM using superquadrics can be used as the base model for dynamics and ergonomics applications with better accuracy because it presents the human motion effectively.
\end{abstract}

Keywords: Superquadric primitive, Levenberg-Marquardt method, Free-Form Deformation, Articulated Human Model

\section{Introduction}

인체의 움직임을 고려한 설계가 중요해짐에 따라 다양한 산업에서 가상인체모델의 필요성이 부각되고 있다. 인체의 거동을 분석하기 위한 가상인체모델은 기구학(kinematic) 및 동역학(kinetics) 이론에 기반하여 실제 인체보다 계산이 용이하도록 단순화시켜 사용된다. 단순화된 가상인체모델은 인체동역학해석에 기반이 되는 모델이며 기본적으로 머리, 팔, 다리 몸통 등의 인체분절요소(body segment)를 조합 하여 생성된다. 인체분절요소는 인체측정학적 데이터베이스 (anthropometric database)로부터 생성될 수 있으며 이때 3 차원 스캐너(scanner) 등 다양한 인체측정장비가 사용된 다. 기존의 관련 연구들은 동역학 해석의 효율성을 높이기
위해 인체분절요소를 타원체(ellipsoid), 실린더(cylinder)와 같은 단순도형 (primitive) 을 사용하여 표현하였다. 그 예로, Chaffin(2001)은 17 개의 분절로 이루어진 가상인체모델을 사용하여 거동범위 분석(reach analysis)에 대한 동적 연구 를 수행하였다. Mikic(2003)은 복셀(voxel) 데이터를 사용 하여 타원체와 원기둥들로 이루어진 인체분절모델을 제안하 였다. 그러나 이러한 연구들은 인체분절요소를 단순화 시켰 기 때문에 각 분절의 관성모멘트 또는 무게중심 등이 실제의 인체와 달리 정의될 수 있다는 한계를 가진다. 따라서 본 연 구에서는 기존의 한계점을 극복하기 위해 superquadric 기 반의 인체분절모델 (Articulated Human Model) 생성방법을 제안하였다. Superquadric이 다양한 형태를 표현할 수 있 는 단순도형이기 때문에 해석의 효율성을 유지함과 동시에 실제 인체에 가깝게 모델링 할 수 있었다. Superquadric으 
로 이루어진 인체분절모델을 생성하기 위해서 인체 분절화 (body segmentation), superquadric 맞춤(fitting) 그리고 자유현상변형 (Free Form Deformation: 이하 FFD)을 이용 하여 오차(error)를 보정하는 단계를 거쳤다.

인체 분절화 단계는 3차원 스캐너로 얻은 데이터(이하 3 차원 전신 스캔 데이터)를 관절(joint)에 따라 17 개의 분 절로 세분화하는 과정이다. 이 과정을 통해 각 부위별 분절 요소를 생성할 수 있었으며 이는 superquadric 맞춤을 하 는데 있어서 기준이 된다. 분절화를 위해 본 연구에서는 관 절의 위치를 기준으로 중력방향과 수직한 방향으로 절단 면(cut plane)을 생성하여 세분화 하였다. 팔의 경우 외전 (abduction)되어 있어 몸통과 평행이 되도록 어깨 관절을 중심으로 회전을 시킨 뒤에 작업하였다. 보행 분석과 같은 경우, 동일한 모델이라 하더라도 관절의 위치에 따라서 결 과 값이 달라지기 때문에 정확한 관절의 위치를 찾는 것 이 중요하다. 따라서 본 연구에서는 각 관절의 위치를 정 확히 찾기 위해 Size Korea에서 제공한 인체측정 데이터와 GEnerator of BOdy Data(GEBOD manual, 1996)에서 제 공하는 관절위치에 대한 연구자료를 이용하였다.

분절화를 통해 얻어진 각각의 분절요소는 superquadric 맞춤을 통하여 타원체보다 기하학적인 정확도가 높은 superquadric으로 표현된다. 여기서 superquadric 맞춤은 분절요 소와 새로이 생성하는 superquadric간의 거리 차를 최소화 (minimization)하는 것을 의미한다. 인체의 형상이 비선형 (non-linear)성을 갖기 때문에 맞춤을 위한 최적화 알고리 즘으로는 Levenberg-Marquard(LM) 방법이 사용되었다. 본 논문에서는 정확한 맞춤을 위해서 분절요소의 무게중심 을 구하여 초기값으로 입력하였으며 각 분절요소의 크기에 맞게 구속조건을 생성하였다. 맞춤의 결과로 superquadric 중심점, 각 축의 길이와 superquadric의 형태를 특징짓는 파라미터(parameter)를 도출할 수 있었다. Superquadric 맞춤으로 도출한 파라미터를 통해 인체모델과 근사한 superquadric 모델이 생성된다. 그러나 LM 방법을 이용한 superquadric 맞춤이 절단면의 불연속적인 부분에서 부정확 하게 표현됨에 따라 형상 보정이 필요하다.

이러한 오차형상을 보정하기 위해 본 논문에서는 $\mathrm{FFD}$ 방 법을 사용하여 superquadric을 변형하였다. $\mathrm{FFD}$ 는 제어점 (control point)를 조절하여 형상을 변형시키는 방법으로 제 어점의 위치는 역 문제(inverse problem)을 통해 구할 수 있었다. Figure 1에서는 본 연구에서 제안하는 인체분절모델 을 생성하기 위한 과정을 나타내었다.

위와 같은 과정을 걸쳐 3 차원 전신 스캔 데이터로부터 생 성된 superquadric으로 이루어진 인체모델은 크게 2가지 장점이 있다. (1) Superquadric 단순도형으로 이루어진 모 델이기 때문에 적은 수의 파라미터만 가지고도 인체형상을

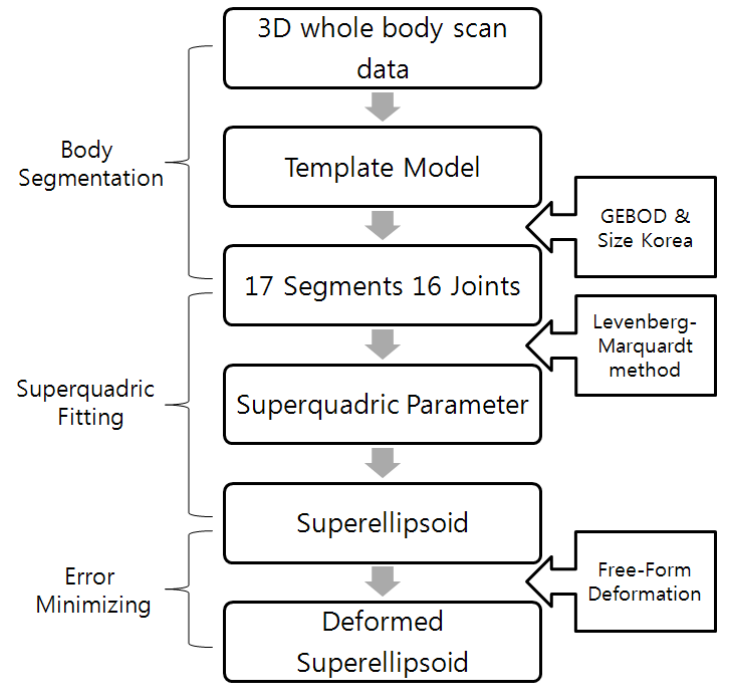

Figure 1. An overview of the method

효과적으로 표현하는 것이 가능하다. 이는 곧 데이터 경량화 로 이어진다. (2) 실제 측정된 데이터를 가지고 인체모델을 생성하였기 때문에 개인 맞춤형 모델을 생성하는데 유용하 게 쓰일 수 있다.

\section{Method}

\subsection{Body segmentation}

인체의 거동을 해석하기 위해서는 인체분절모델이 필 요하며 이를 위해서는 인체 분절화 과정을 거쳐야 한다. 본 논문에서의 인체 분절화는 3 차원 전신 스캔 데이터를 superquadric 맞춤에 사용될 수 있도록 인체분절요소로 세 분화시키는 단계를 의미한다. 사용된 3 차원 전신 스캔 데이 터는 키 $174 \mathrm{~cm}$ 에 몸무게 $65 \mathrm{~kg}$ 인 표준체형을 가진 20세 한국인 남성을 3 차원 스캐너를 통해 측정한 것이다. 3 차원 스캐너는 인체형상을 매우 사실적으로 표현할 수 있으며 점 (vertex)정보와 면(face)정보를 가지고 있는 PoLYgon file format(PLY)을 제공한다. 그러나 3차원 스캐너의 특성상 손, 발바닥, 겨드랑이 등의 부분은 정확한 스캔이 어려워 이 에 따른 형상 보정이 필요하다.

\subsubsection{Preprocessing}

본 연구에서는 정확한 superquadric 맞춤 결과 값을 얻기 위해 데이터 포맷을 변환하고 3차원 전신 스캔 데이터가 표 현하는 가상인체모델의 형상을 변형하였다. 여기서 데이터 포맷의 변환이란 PLY 포맷의 인체 데이터(3차원 전신 스 
캔 데이터)를 솔리드 모델(solid model)을 표현할 수 있는 $\mathrm{STEP}$ 포맷으로 변환하는 과정을 의미한다.

솔리드 모델을 얻기 위해 우선 PLY 포맷의 인체 데이터 를 가공이 간편한 단순 점 데이터로 이루어진 STL 포맷으 로 변환하였다. STL 포맷으로 변환된 인체 데이터는 곡면생 성 (surfacing) 과정을 거쳐 서피스 모델로 생성할 수 있다. 서피스 모델은 CATIA V5에서 최종적으로 솔리드 모델로 변환이 가능하다. 본 연구에서 사용된 가상인체모델의 경우 손가락 부분이 부정확하게 표현되어 있어 손을 Figure 2 와 같이 단순화시켰다. 인체분절요소의 회전을 고려하지 않도록 하기 위해 외전된 팔을 어깨 관절을 기준으로 회전시켜 몸통 과 수평이 되도록 하였다. 이러한 파일 포맷변환 작업과 형상 보완 작업을 끝낸 솔리드 모델은 superquadric 맞춤을 할 때 기준이 되기 때문에 타겟모델(Target model)이라 한다.

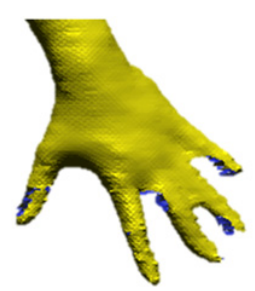

(a)

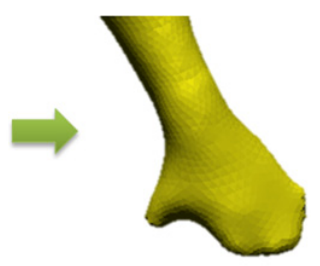

(b)
Figure 2. Left hand smoothing (a) low data (b) smoothed data

\subsubsection{Find joint locations}

인체의 관절위치를 기준으로 분절화 하기 위해 가장 중요 한 것은 정확한 인체관절위치를 찾는 것이다. 그러나 타겟모 델의 경우 단순히 인체의 외형만을 나타내고 있기 때문에 정 확한 인체관절위치를 찾기가 어렵다. 본 논문에서는 미국에 서 군사용 목적으로 개발한 $\mathrm{GEBOD}$ 프로그램의 연구내용을 바탕으로 관절위치를 찾아내었다.

본 논문에서는 표준 남성체형 모델을 가지고 타겟모델을 생성하였으므로 GEBOD의 Adult Human Male 타입에서 사용된 방법을 이용하였다(Appendix 1). 이는 관절위치를 찾기 위해 회귀 방정식을 사용하는 방법으로 인체 기준점의 위치좌표를 필요로 한다. 관절의 위치를 찾는 것과는 달리 기준점은 인체측정에 필요한 기준이 되는 점이기 때문에 대 부분 눈으로 보거나 손으로 만져서 찾을 수 있으며 인체피 부에 표기가 가능하다. 기준점에 대한 데이터는 Size Korea 에서 총 70 개(머리 부위 10 개, 목 부위 4 개, 몸통 부위 27 개, 다리 부위 13 개, 발 부위 4 개, 팔 부위 9 개, 및 손 부위 3 개)를 제공받았다. 70 개의 기준점 중 관절의 위치를 찾는 데 필요한 28 개의 기준점을 가지고 16 개의 관절의 위치를
찾을 수 있었다.

\subsubsection{Body split}

타겟모델을 생성하였고 절단할 때 기준이 되는 관절의 위 치를 구했기 때문에 분절화가 가능하다. 본 연구에서는 분절 화를 위해 절단면을 생성하였으며, 대부분의 절단면은 XY 평면에 생성할 수 있었다. 이때 Z좌표 (중력방향이 -방향) 값은 관절위치로부터 구할 수 있다. 분절화에 사용된 모델은 팔이 몸으로부터 약 $24^{\circ}$ 만큼 외전되어 있기 때문에 절단면 을 XY 평면으로 생성할 경우 superquadric 맞춤의 결과가 부정확하게 나오게 된다. 따라서 어깨 관절을 중심으로 회전 시켜 팔을 지면과 수직이 되도록 하였다. 지면과 수직방향을 이루게 된 팔은 어깨 관절을 중심으로 다른 부분과 달리 XZ 평면에 절단면을 생성한다. 다리와 몸 사이의 절단면의 경우 도 보다 정확한 superquadric 맞춤 결과를 얻기 위해 관절 을 중심으로 $45^{\circ}$ 기울어 생성하였다. 각 관절에 따라 절단면 을 생성할 수 있기 때문에 총 16 개의 절단면을 생성할 수 있었으며 16 개의 절단면에 의해 최종적으로 17 개의 분절요 소가 생성되었다. 이는 기존의 방법(Park, 1999)과 유사하며 최종적으로 타겟모델을 세분화 한 결과는 Figure 3과 같다.

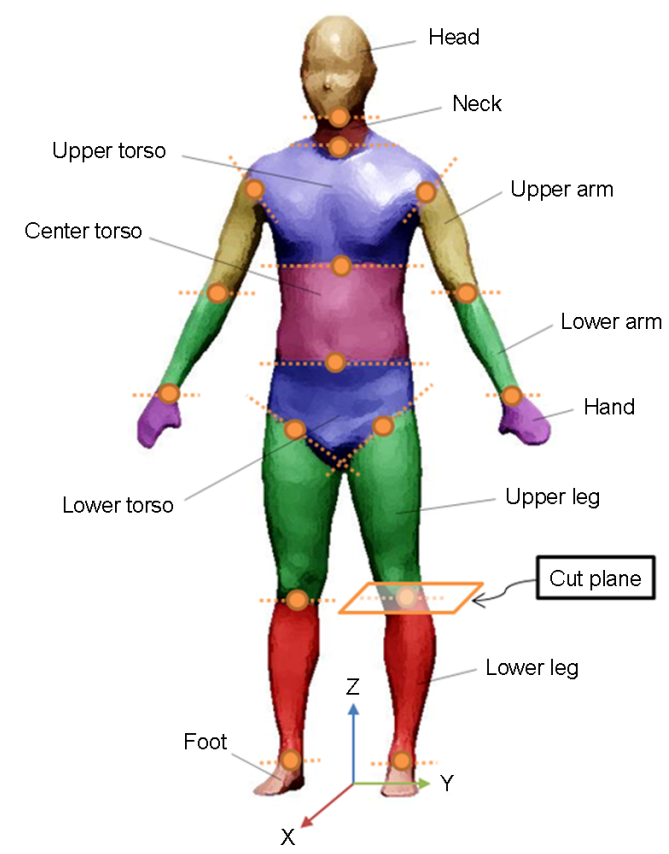

Figure 3. Segmented target model(17 segments, 16 joints)

\subsection{Superquadric fitting}

\subsubsection{Definition of superquadric}

Superquadric은 다양한 형상을 표현가능하고 3차원의 
표현 수식이 비교적 간단하다. 또한, 3차원 상에서의 집합 연산에 사용되는 함수가 수학적으로 정의되므로 물체의 표 현과 인식에 매우 유용하게 사용된다. 본 논문에서는 이러한 superquadric을 기반으로 인체의 각 분절을 대표하는 분절 형상을 표현하고자 하였다. Superquadric은 형상에 따라 크 게 4 가지로 분류할 수 있으며 그 중 인체의 형상을 가장 효 과적으로 표현할 수 있는 superellipsoid를 사용하였다.

Superellipsoid를 정의하는 음함수(implicit function) 표 현은 식 1 과 같으며, superellipsoid의 형상을 표현하기 위 한 음함수 방정식은 안-바깥(inside-outside) 함수로 나타 낼 수 있다.

$\left(\left(\left(\frac{\mathrm{x}}{\mathrm{a}_{1}}\right)^{2 / \varepsilon_{2}}+\left(\frac{\mathrm{y}}{\mathrm{a}_{2}}\right)^{2 / \varepsilon_{2}}\right)^{\varepsilon_{2} / \varepsilon_{1}}+\left(\frac{\mathrm{z}}{\mathrm{a}_{3}}\right)^{2 / \varepsilon_{1}}\right)^{\varepsilon_{1} / 2}=1$

$\left(\left(\left(\frac{\mathrm{x}}{\mathrm{a}_{1}}\right)^{2 / \varepsilon_{2}}+\left(\frac{\mathrm{y}}{\mathrm{a}_{2}}\right)^{2 / \varepsilon_{2}}\right)^{\varepsilon_{2} / \varepsilon_{1}}+\left(\frac{\mathrm{z}}{\mathrm{a}_{3}}\right)^{2 / \varepsilon_{1}}\right)^{\varepsilon_{1} / 2}=1$

$\mathrm{F}(\mathrm{x}, \mathrm{y}, \mathrm{z})=1,(\mathrm{x}, \mathrm{y}, \mathrm{z})$ is on the surface,

$\mathrm{F}(\mathrm{x}, \mathrm{y}, \mathrm{z})<1,(\mathrm{x}, \mathrm{y}, \mathrm{z})$ is inside,

$\mathrm{F}(\mathrm{x}, \mathrm{y}, \mathrm{z})>1,(\mathrm{x}, \mathrm{y}, \mathrm{z})$ is outside.

파라미터 $\varepsilon_{1}, \varepsilon_{2}$ 에 따른 superellipsoid 형태는 Figure 4 와 같다. $\varepsilon_{1}, \varepsilon_{2}$ 이 0에 근접할수록 부피가 커지며 직사각형

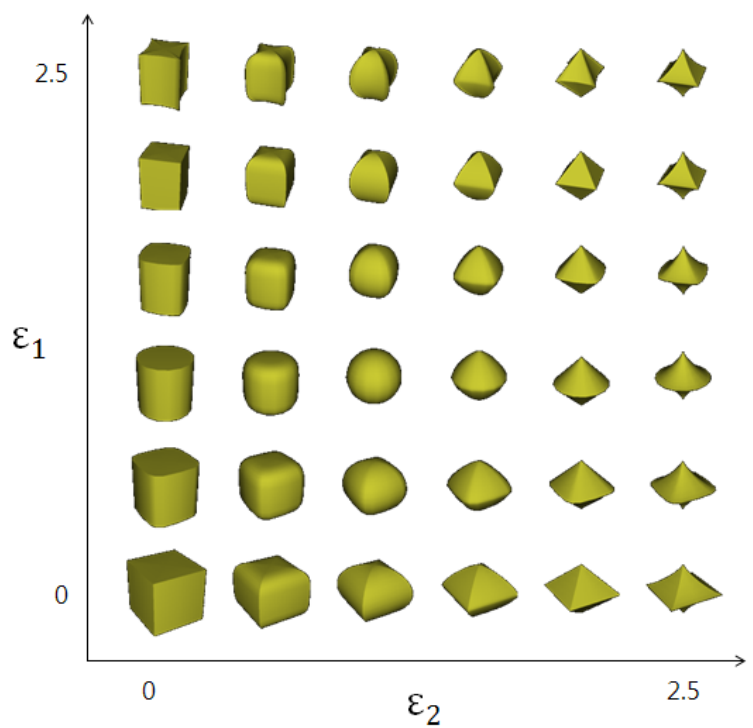

Figure 4. Examples of superellipsoids according to $\varepsilon_{1}$ and $\varepsilon_{2}$
에 가깝고 $\varepsilon_{1}, \varepsilon_{2}$ 이 커질수록 전체적인 부피가 감소함을 볼 수 있다. 또한 $\varepsilon_{1}$ 이 $1, \varepsilon_{2}$ 가 0이면 실린더 형태를 띠는 것을 볼 수 있다.

\subsubsection{Fitting method}

본 논문에서는 최소자승법 (Least-Squares Method)을 통해 superquadric 맞춤을 하였다(Solina, 1990). Solina 가 사용한 파라미터는 총 11 개이며 superellipsoid의 형태 를 결정짓는 5 개의 파라미터 외에도 이동행렬 (translation matrix) 과 오일러 각(Euler angle: $\varphi, \theta, \psi$ )을 결정짓는 6 개의 파라미터가 추가되었다. 따라서 확장된 안-바깥 함 수는 11 개의 파라미터를 가진다. 11 개의 파라미터를 제대 로 표현하려면 다음과 같이 프레임 변환(Frame change) 을 거쳐야 한다. 프레임 변환을 하기 위해 필요한 변환행렬 (transform matrix: T) 을 구하면 다음과 같다.

$$
\mathrm{T}=\left[\begin{array}{ll}
\mathrm{R} & \mathrm{t} \\
0 & 1
\end{array}\right]
$$

위 행렬에서 $\mathrm{R}$ 은 $3 \times 3$ 회전행렬(rotation matrix)이고, $\mathrm{t}$ 는 $3 \times 1$ 변환벡터(translation vector)이다. $\mathrm{T}$ 를 통해 최소 화 알고리즘(minimization algorithm)에 사용될 수 있는 함 수(식 4)를 유도할 수 있다.

$$
\hat{\mathrm{F}}(\underline{\mathrm{x}})=\mathrm{F}\left(\mathrm{T}^{-1}(\underline{\mathrm{x}})\right)
$$

식 4 을 최소자승법에 대입하면 다음과 같다.

$E(A)=\min \sum_{i=1}^{N}\left[\sqrt{a_{1} a_{2} a_{3}}\left(1-\hat{F}\left(a_{1}, a_{2}, a_{3}, \varepsilon_{1}, \varepsilon_{2}, \varphi, \theta, \psi, t_{x}, t_{y}, t_{z}\right)\right]^{2}\right.$

식 5 의 해를 도출함으로써 인체분절요소와 superellipsoid 평면간의 거리를 최소화하는 파라미터 값을 얻을 수 있다. 인체의 형상이 비선형성을 띠기 때문에 식 5 는 비선형 최소 자승법인 LM 방법을 통해 얻어진 것이다. 본 연구에서는 지 면과 수직으로 이루고 있는 인체분절요소를 사용하였으므로 회전을 고려하지 않고 superquadric 맞춤을 수행하였다. 따 라서, superquadric 맞춤을 통해 도출되는 파라미터는 오일 러 각 파라미터를 제외한 8개가 된다.

최소자승법의 해를 구할 때 초기값(initial condition)과 구속조건(constraint)을 형상에 대한 고려 없이 설정하면 전체 최소값(global minimum)이 아닌 국부 최소값(local minimum)을 찾게 되어 의도되지 않은 해 값에 수렴하게 된 다. 따라서 정확한 결과 값을 도출하려면 맞추려는 인체분 
Table 1. Fitted superquadric parameter using levenberg-marquardt method

\begin{tabular}{|c|c|c|c|c|c|c|c|c|c|c|c|}
\hline & \multicolumn{3}{|c|}{ Initial condition } & \multicolumn{8}{|c|}{ Parameter results } \\
\hline & $\mathrm{G}_{\mathrm{x}}$ & $\mathrm{G}_{\mathrm{y}}$ & $\mathrm{G}_{\mathrm{z}}$ & $t_{1}$ & $\mathrm{t}_{2}$ & $t_{3}$ & $a_{1}$ & $a_{2}$ & $a_{3}$ & $\varepsilon_{1}$ & $\varepsilon_{2}$ \\
\hline Head & 66.28 & 2.77 & 1606.53 & 65.56 & 1.84 & 1601.99 & 91.31 & 74.36 & 146.73 & 1.00 & 0.82 \\
\hline Neck & 21.13 & 1.10 & 1469.65 & 25.98 & -0.40 & 1428.07 & 44.72 & 36.84 & 100.00 & 1.00 & 0.53 \\
\hline Upper Torso & -0.61 & -2.41 & 1289.66 & 0.00 & 0.00 & 1278.45 & 110.42 & 186.57 & 180.00 & 1.00 & 0.54 \\
\hline Central Torso & 7.81 & -4.50 & 1060.31 & 9.60 & -4.66 & 1055.86 & 106.84 & 152.55 & 180.00 & 0.96 & 0.75 \\
\hline Lower Torso & -13.63 & -18.01 & 898.19 & -14.94 & -5.93 & 888.93 & 130.46 & 162.86 & 173.81 & 1.00 & 0.90 \\
\hline Right Upper Arm & -2.35 & -207.24 & 1177.80 & -0.17 & -223.62 & 1217.60 & 44.85 & 61.57 & 242.37 & 1.00 & 0.52 \\
\hline Right Lower Arm & -1.45 & -167.10 & 1118.79 & 7.51 & -167.72 & 1022.16 & 46.56 & 39.23 & 162.14 & 1.00 & 0.66 \\
\hline Right Hand & 4.23 & -171.81 & 792.12 & 6.31 & -202.23 & 857.80 & 11.33 & 103.42 & 34.57 & 1.00 & 0.14 \\
\hline Left Upper Arm & -2.53 & 209.04 & 1183.18 & -0.06 & 222.92 & 1218.40 & 44.35 & 60.85 & 240.12 & 1.00 & 0.51 \\
\hline Left Lower Arm & -1.05 & 169.40 & 1117.79 & 7.21 & 168.72 & 1002.16 & 46.76 & 39.30 & 160.14 & 1.00 & 0.61 \\
\hline Left Hand & 4.13 & 174.71 & 793.10 & 6.11 & 200.53 & 860.80 & 12.19 & 100.00 & 33.87 & 1.00 & 0.13 \\
\hline Right Upper Leg & -33.81 & -94.49 & 592.74 & -25.62 & -84.93 & 703.25 & 101.56 & 79.40 & 301.79 & 1.00 & 0.69 \\
\hline Right Lower Leg & -85.31 & -121.40 & 305.53 & -79.86 & -114.96 & 347.60 & 66.82 & 53.48 & 267.50 & 1.00 & 0.82 \\
\hline Right Foot & -19.21 & -117.93 & 23.52 & -21.23 & -120.08 & 33.93 & 104.55 & 41.00 & 46.43 & 1.00 & 0.52 \\
\hline Left Upper Leg & -33.01 & 93.49 & 593.39 & -27.72 & 87.81 & 707.18 & 100.83 & 81.40 & 300.23 & 1.00 & 0.67 \\
\hline Left Lower Leg & -86.91 & 120.10 & 303.93 & -78.66 & 115.66 & 349.40 & 67.62 & 54.38 & 269.60 & 1.00 & 0.83 \\
\hline
\end{tabular}

절요소의 크기를 고려하여 초기값과 구속조건을 설정해주 어야 한다. 본 연구에서는 인체분절요소의 무게중심 $\left(G_{x}, G_{y}\right.$, $\mathrm{G}_{z}$ )을 $\mathrm{t}_{1}, \mathrm{t}_{2}, \mathrm{t}_{3}$ 의 초기값으로 설정하였으며 절단면에서 측 정한 길이의 평균을 $\mathrm{a}_{1}, \mathrm{a}_{2}, \mathrm{a}_{3}$ 의 초기값으로 설정하였다. $\varepsilon_{1}, \varepsilon_{2}$ 의 초기값은 1 로 하였으며 이는 타원체에 해당한다. Superquadric 맞춤을 통하여 도출한 파라미터 값은 Table 1 에 나타내었다.

타겟모델을 세분화한 뒤 불연속 구간인 절단면 부분의 형 상을 보정하지 않았기 때문에 맞춤 결과를 보면 절단면에서 오차가 크게 발생함을 알 수 있다. 종아리의 경우 위쪽 부분 의 부피가 더 크기 때문에 맞춤을 하면 실제 종아리보다 솟 아오른 형태를 띠는 superellipsoid가 생성된다. Figure 5에 왼쪽 종아리의 맞춤 결과를 나타내었으며 맞춤 시 오차가 생 기는 방향을 파란색으로 표기하였다. 맞춤 오차를 보완하기 위한 방법으로 인체분절요소를 맞춤하기 전에 미리 가공하 여 좀 더 정확한 맞춤 결과 값을 도출하는 방법과 맞춤을 통 하여 생성된 superellipsoid를 다시 인체분절요소에 맞게 변 형을 시키는 방법이 있다. 기존의 연구(Bardinet, 1994)에 서 superquadric의 단점을 $\mathrm{FFD}$ 를 통하여 보완한 것을 착 안하여 본 연구에서는 superellipsoid를 변형시켜 오차를 최 소화 하였다.

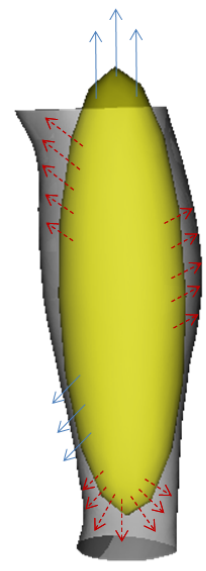

(a)

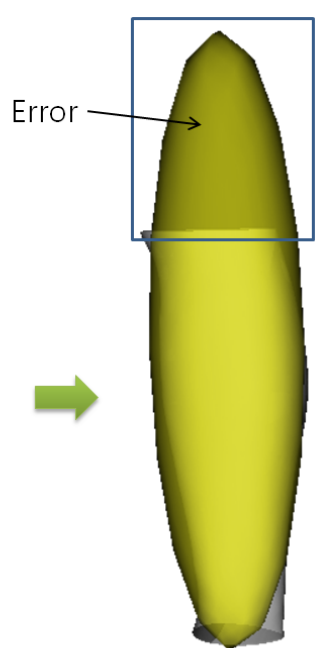

(b)
Figure 5. Fitting result of left lower leg (a) Before fitting(with initial condition) (b) After fitting

\subsection{Error minimization using Free-Form Deformation}

본 절에서는 $\mathrm{FFD}$ 를 이용해서 어떻게 error를 최소화했는 지를 기술하고자 한다. 따라서, $\mathrm{FFD}$ 에 대한 간단한 정의와 
함께 error를 최소화 하는 값(제어점 위치)을 구하기 위해 사용된 역 문제에 대한 해결 방법을 설명하고자 한다.

\subsubsection{Definition of Free-Form Deformation}

$\mathrm{FFD}$ 는 물체의 표현방식에 관계없이 다양한 물체의 변형 을 효과적으로 생성할 수 있는 방법으로, 컴퓨터 그래픽스에 서 가장 널리 사용되는 변형기법 중의 하나이다. 이 방법은 직육면체들로 이루어진 3차원 제어격자(control lattice)를 물체를 포함하는 공간상에 배치하고, 이 제어격자의 제어점 (control point)을 조작하여 물체를 변형하는 방법이다.

$\mathrm{FFD}$ 의 장점은 물체의 형상에 관계 없이 전체적 혹은 국 소적으로 변형이 가능하다는 점과 곡선과 곡면의 파라미터 가 변형 후에도 여전히 유지된다는 점이다. 이는 인체의 모 든 분절요소를 변형시킬 수 있다는 것을 의미함과 동시에 변형 후에도 superellipsoid를 정의하는 식이 그대로 보존됨 을 의미한다. 위와 같은 장점 때문에 본 논문에서는 $\mathrm{FFD}$ 를 이용하여 오차를 보정하였다.

$\mathrm{FFD}$ 를 수행하기 위해서는 제어격자의 변화량에 간단한 수학적인 함수를 적용하여 물체를 포함한 공간 전체에서 정 의되는 변형함수를 구한 뒤, 이 변형함수를 물체상의 점에서 계산하여 변형된 물체의 형태를 구해야 한다. 여기서 물체를 내포하는 제어격자는 한 점 $\mathrm{X}_{0}$ 와 직교(orthogonal)한 벡터 $\mathrm{S}, \mathrm{T}$ 와 $\mathrm{U}$ 를 가지고 정의되며, 임의의 한 점 $\mathrm{X}$ 는 $\mathrm{X}_{0}, \mathrm{~S}, \mathrm{~T}$ 와 U로 정의되는 local 좌표계에 의해서 다음과 같이 나타 낼 수 있다.

$$
\mathrm{X}=\mathrm{X}_{0}+\mathrm{sS}+\mathrm{tT}+\mathrm{uU}
$$

선형대수학의 개념을 이용하면 X의 (s, t, u) 좌표 값은 다음과 같이 쉽게 구할 수 있다.

$$
\begin{aligned}
& \mathrm{s}=\frac{\mathrm{T} \times \mathrm{U} \cdot\left(X-X_{0}\right)}{\mathrm{T} \cdot \mathrm{U} \times \mathrm{S}}, \mathrm{t}=\frac{\mathrm{S} \times \mathrm{U} \cdot\left(X-X_{0}\right)}{\mathrm{S} \cdot \mathrm{U} \times \mathrm{T}}, \mathrm{u}=\frac{\mathrm{S} \times \mathrm{T} \cdot\left(X-\mathrm{X}_{0}\right)}{\mathrm{S} \cdot \mathrm{T} \times \mathrm{U}} \\
& \text { 이때, } 0 \leq \mathrm{s} \leq 1, \quad 0 \leq \mathrm{t} \leq 1, \quad 0 \leq \mathrm{u} \leq 1
\end{aligned}
$$

$\mathrm{S}, \mathrm{T}, \mathrm{U}$ 방향으로 각각 $1+1, m+1, n+1$ 개의 격자구조 (grid)를 생성한다면, $\mathrm{P}_{\mathrm{ijk}}$ (control points)는 다음과 같이 표 현된다.

$$
\mathrm{P}_{\mathrm{ijk}}=\mathrm{X}_{0}+\frac{\mathrm{i}}{l} \mathrm{~S}+\frac{\mathrm{j}}{m} \mathrm{~T}+\frac{\mathrm{k}}{n} \mathrm{U}
$$

제어점 $\mathrm{P}_{\mathrm{ijk}}$ 를 원하는 위치로 이동함으로써 물체의 변형이 일어나고, $\mathrm{X}$ 의 변형된 점 $\mathrm{X}_{\mathrm{ffd}}$ 는 다음과 같이 표현된다.

$$
\begin{aligned}
& \mathrm{X}_{\mathrm{ffd}}=\sum_{\mathrm{i}=0}^{l} \mathrm{~B}_{\mathrm{i}}^{l}(\mathrm{~s}) \sum_{\mathrm{j}=0}^{m} \mathrm{~B}_{\mathrm{j}}^{m}(\mathrm{t}) \sum_{\mathrm{k}=0}^{n} \mathrm{~B}_{\mathrm{k}}^{n}(\mathrm{u}) \mathrm{P}_{\mathrm{ijk}} \\
& \mathrm{B}_{\mathrm{i}}^{l}(\mathrm{~s})=\left(\begin{array}{c}
l \\
\mathrm{i}
\end{array}\right)(1-\mathrm{s})^{(l-\mathrm{i})} \mathrm{s}^{\mathrm{i}} \\
& \mathrm{B}_{\mathrm{j}}^{m}(\mathrm{t})=\left(\begin{array}{c}
m \\
\mathrm{j}
\end{array}\right)(1-\mathrm{t})^{(m-\mathrm{j})} \mathrm{t}^{\mathrm{j}} \\
& \mathrm{B}_{\mathrm{k}}^{n}(\mathrm{u})=\left(\begin{array}{c}
n \\
\mathrm{k}
\end{array}\right)(1-\mathrm{u})^{(n-\mathrm{k})} \mathrm{u}^{\mathrm{k}}
\end{aligned}
$$

식 9가 trivariate Bernstein polynomial의 텐서곱(tensor product)으로 정의되므로, 결과적으로 변형된 superellipsoid 는 B-spline 형태의 곡면을 띠게 된다.

\subsubsection{The inverse problem}

$\mathrm{FFD}$ 를 사용하여 보정을 하려면 인체분절요소와 superquadric 맞춤을 통해 생성되었던 superellipsoid간의 거리 를 최소화 시키는 제어점의 위치를 계산할 수 있어야 한다. W.M.Hsu(1992)는 의사역 방법(pseudoinverse)을 이용 하여 제어점의 위치를 계산하는 방법을 제안하였다. 역 문 제의 특징은 유일해가 존재하지 않고 부족조건식 (underdetermined)으로 존재한다는 것이다. 따라서 보다 수월하게 문제를 해결하기 위해서는 조절하고자 하는 제어점의 개수 를 제한해야 할 필요가 있다.

역 문제를 해결하기 위해서는 우선 인체분절요소와 superellipsoid의 유클리드 거리(Euclidean distance)를 계산하여 인체분절요소를 이루는 점 중에 superellipsoid에 가장 가까 운 점(closest point)을 찾아야 한다. 그 다음 superellipsoid 가 그에 맞춰지도록 하는 제어점의 새로운 위치를 찾음으로 마무리된다. FFD의 결과로 얻어진 새로운 superquadric의 위치를 $\mathrm{X}$, 제어점의 위치를 $\mathrm{P}_{\mathrm{ijk}}$, 제어점과 이에 상응하는 superellipsoid과의 외적을 B라하면 다음과 같은 형태의 식 을 얻을 수 있다.

$$
\mathrm{X}=\mathrm{BP}
$$

제어점의 위치를 찾는 방법은 크게 정규 방정식(Normal equation) 과 특이 값 분해 (Singular Value Decomposition: 이하 SVD) 가 있다. 우선 정규 방정식에 대해 알아보면 정규 방정식은 Conjugate Gradient Method를 이용하여 해를 찾 는 것으로 식 10 을 $\mathrm{B}^{\mathrm{T}} \mathrm{X}=\left(\mathrm{B}^{\mathrm{T}} \mathrm{B}\right) \mathrm{P}$ 로 변환하여 구한다. 따라 서 $\mathrm{P}$ (제어점 위치)를 구하는 식은 다음과 같다.

$$
P=\left(B^{T} B\right)^{-1} B^{T} X
$$

$\mathrm{SVD}$ 는 선형대수학에서 나오는 이론으로 $\mathrm{B}^{-1}$ 를 diagonal 
matrix $(\Sigma)$, orthogonal matrix $(\mathrm{V})$ 와 column-orthogonal $\operatorname{matrix}(\mathrm{U})$ 로 성분 분해하여 다음과 같이 해 값을 찾는다.

$$
\mathrm{P}=\mathrm{U} \sum \mathrm{V}^{\mathrm{T}} \mathrm{X}
$$

식 11 혹은 식 12 를 통해 구한 $\mathrm{P}_{\mathrm{ijk}}$ 를 가지고 식 9 에 대 입하면 오차를 최소화 시키는 $\mathrm{FFD}$ 결과를 얻을 수 있다. 왼 쪽 종아리 부위의 보정된 결과는 Figure 6과 같으며 오차가 확연히 줄어든 것을 볼 수 있다. 이러한 superquadric 맞춤 및 오차 보정(error minimization) 과정을 모든 인체분절요 소에 걸쳐서 수행함으로써 완전한 인체분절모델을 생성하 였다.

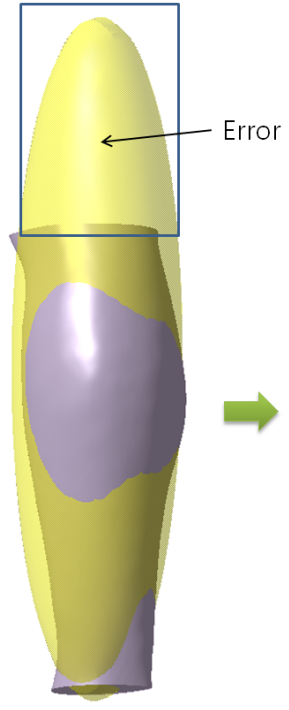

(a) (b)

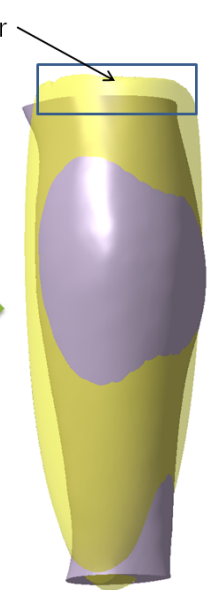

Figure 6. Error minimization result of left lower leg (a) Before deformation(fitted superellipsoid) (b) After deformation

\section{Results \& Discussion}

본 논문에서는 3 차원 전신 스캔 데이터를 사용하여 17 개 의 superellipsoid와 16 개의 관절 조인트로 이루어진 인체 분절모델을 생성하였다. 이를 구현하기 위해 인체 분절화, superquadric 맞춤 그리고 오차 보정을 수행하였다. 분절화 단계에서는 3 차원 전신 스캔 데이터를 사용하여 타겟모델을 생성하고 이를 각 관절에 따라 세분화 함으로써 인체분절요 소를 얻을 수 있었다. Superquadric 맞춤 단계에서는 LM 방 법을 사용하여 인체형상에 맞는 superquadric 파라미터 값 을 도출할 수 있었으며, 이 값을 가지고 superellipsoid를 생
성하기 위해서 상용 그래픽 라이브러리인 Visualization Tool $\mathrm{Kit}(\mathrm{VTK})$ 을 사용하였다. 오차 보정 단계에서는 superquadric 맞춤을 수행하면서 생긴 오차 부분을 $\mathrm{FFD}$ 를 이용 하여 보정하였다. 최종적으로 완전한 인체분절모델을 생성하 기 위해 각 인체분절마다 superquadric 맞춤과 $\mathrm{FFD}$ 를 통 한 오차 보정을 하였다. 맞춤한 결과 값을 살펴보면 대부분 의 인체분절요소에서 $\varepsilon_{1}$ 파라미터가 1 인 것을 볼 수 있었 다. 이는 인체형상이 타원체에 가깝기 때문인 것으로 판단된 다. 손과 발 부분에서 맞춤의 결과가 좋지 않았는데 그 이유 는 손과 발 부분의 형상이 다른 인체분절요소에 비해 복잡한 형상을 띠었기 때문으로 분석된다. 또한, 절단면이 넓을수록 절단면에서 생기는 오차가 커짐을 알 수 있었는데 이를 보정 하기 위하여 $\mathrm{FFD}$ 를 이용하였다. 본 논문에서 제안한 모델은 단순도형으로 이루어진 모델이기 때문에 데이터 용량을 타 겟모델의 약 $43 \%$ 로 간소화 시킬 수 있었다(target model: 22.516KB, superquadric model: 9.84KB).

19 개의 인체분절요소와 16 개의 관절로 이루어진 기존의 타원체 인체분절모델(Lee, 2002)을 살펴보면 타원체 형상 의 한계 때문에 타원체가 연결되는 부분(인체관절 부분)에 서 실제 인체와 형상차이가 크게 나타남을 알 수 있다. 타원 체와 달리 superellipsoid을 사용하면 구에서부터 실린더에 이르기까지 다양한 형상을 표현할 수 있으므로 인체관절 부 분도 비교적 정확히 표현이 가능하다. 정확한 검증을 위해 각 인체모델의 무게중심(Table 2) 과 표면적 (Table 3)을 비 교한 결과 superellipsoid 인체분절모델이 타원체 인체분절 모델보다 타겟모델에 더 근사함을 알 수 있었다. Figure 7는 center torso를 타원체와 superellipsoid를 사용하여 fitting 한 결과를 비교한 것으로 타원체 보다 superellipsoid가 인 체를 더 효과적으로 표현함을 볼 수 있다. 이는 단순히 형상 이 보다 사실적 이다는 것만 의미하는 것이 아니라 동역학 해석 시 더 정확한 운동해석이 가능하다는 것까지 내포한다. 각각의 인체모델의 전체 형상을 비교하면 Figure 8과 같다.

Table 2. Comparison of the center of mass in left lower leg

\begin{tabular}{c|c|c|c}
\hline & $\mathrm{G}_{\mathrm{x}}$ & $\mathrm{G}_{\mathrm{y}}$ & $\mathrm{G}_{\mathrm{z}}$ \\
\hline Target & -86.91 & 120.10 & 303.93 \\
\hline Superellipsoid & -82.49 & 116.53 & 302.54 \\
\hline Ellipsoid & -69.69 & 118.96 & 238.16 \\
\hline
\end{tabular}

Table 3. Comparison of the area in left lower leg

\begin{tabular}{c|c|c|c}
\hline & Target & Ellipsoid & Superellipsoid \\
\hline Area $\left(\mathrm{m}^{2}\right)$ & 0.23 & 0.181 & 0.221 \\
\hline
\end{tabular}




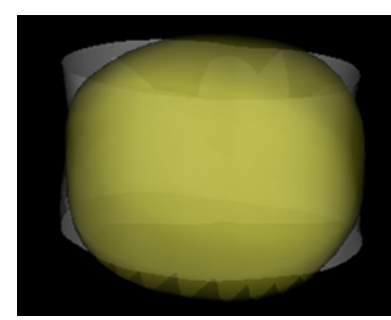

(a)

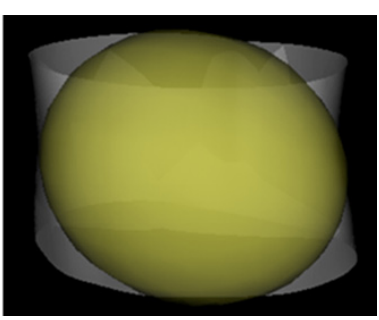

(b)
Figure 7. Comparison of superellipsoid and ellipsoid (a) superellipsoid (b) ellipsoid

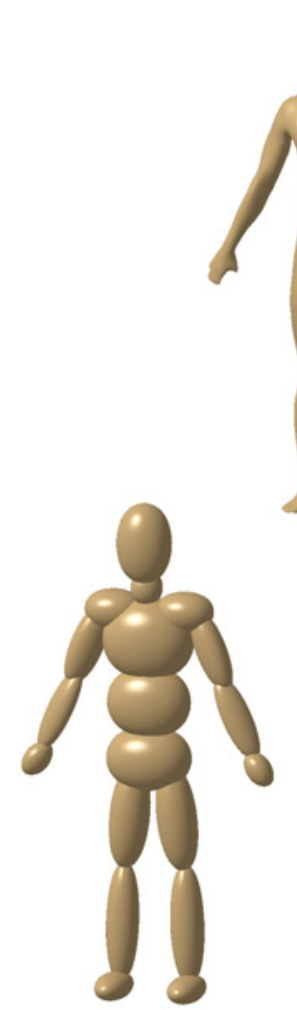

(b)

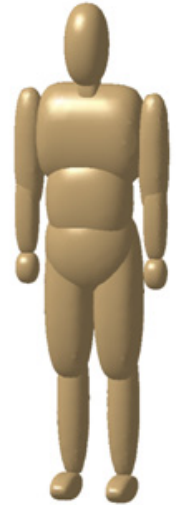

(c)
Figure 8. Human model (a) target model (b) AHM with ellipsoids (c) AHM with superellipsoids

\section{Conclusion}

본 논문은 인체의 형상과 움직임을 정확히 나타내면서도 간소한 형태로 인체를 표현할 수 있는 가상인체모델을 생성 하는 방법을 제안하였다. 이를 위해 타원체보다 인체의 형상 을 정확하게 표현할 수 있는 superquadric을 이용하여 인체 모델을 생성하였다. Superquadric으로 인체형상을 표현하려
면 맞춤을 하기 위한 기준이 되는 모델이 필요하며 이를 위 해 본 연구에서는 20 세 표준체형 남자의 3 차원 전신 스캔 데이터를 이용하였다. 인체분절요소에 따라 superquadric을 생성하기 위해 각 관절을 중심으로 분할하여 17 개로 세분화 하였다. 이때 정확한 관절의 위치를 찾기 위해 $\mathrm{GEBOD}$ 의 연구자료를 참조하였다. 인체분절요소와 superquadric 간의 거리를 최소화하는 superquadric 파라미터를 구하기 위해 $\mathrm{LM}$ 방법이 사용되었으며 맞춤을 하면서 생긴 오차는 $\mathrm{FFD}$ 을 사용하여 최소화 하였다. 본 연구에서는 3 차원 전신 스캔 데이터를 이용하였기 때문에 실제 인체와 유사한 모델을 얻 을 수 있으며, 이는 개인 맞춤형 모델을 제작하는데 밑바탕 이 된다. 그러나 3 차원 전신 스캔 데이터 없이는 인체분절모 델을 생성하기 어렵다는 한계점이 있다. 추후에 인체형상에 따른 superquadric 파라미터의 경향성을 파악한다면 스캔 데이터 없이 다양한 인체형상을 표현할 수 있는 인체분절모 델을 생성할 수 있을 것이다.

본 연구에서 생성한 인체분절모델의 특징은 다음과 같이 요약할 수 있다. (1) 인체형상정보를 기반으로 생성된다. (2) 17 개의 인체분절요소가 각기 다른 형상의 superellipsoid로 표현된다. (3) 동역학 해석 시 필요한 16 개의 관절위치 정보 를 가지고 있다. (4) 8개의 superquadric 파라미터 값만 가지고 인체모델 구현이 가능하다.

\section{Acknowledgements}

This work was supported by the Research fund of Survivability Technology Defense Research Center of Agency for Defense Development of Korea(No. UD090090GD).

\section{References}

Bardinet, E., et al. "Fitting of Iso-Surfaces Using Superquadrics and FreeForm Deformation", the proceedings of the IEEE WBIA, 184-193, 1994.

Chaffin, D. B., "Digital Human Modeling for Vehicle and Workplace Design", SAE International, 2001

Cheng, H., et al. Generator of Body Data Manual, NTIS, Springfield, 1994.

Cheng, H., et al. "The Development of the GEBOD Program", Proceedings of the 15h Biomedical Engineering Conference, (pp.251-254), Dayton. OH. USA. 1996.

Danielsson, P. E., "Euclidean Distance Mapping", Computer Graphics and 
Image Processing, 14(3), 227-248, 1980.

Esteves, G., et al. "Development of a Model of the Muscle Skeletal System using Adams", SAE International, 2004-01-2169, 2004.

Lee, S. C., et al. "Development and Application of Korean Dummy Models", Journal of the Ergonomics Society of Korea, 21(2), 13-23, 2002.

Marquez, J., et al. "Robust Ellipsoid Model Fitting of Human Heads", Lecture Notes in Computer Science, 4931/2008, 381-390, 2008.

Mikic, I., et al. "Human Body Model Acquisition and Tracking Using Voxel Data", International Journal of Computer Vision, 53(3), 199 $-223,2003$.

Park, S. J., et al. "Anthropometric and Biomechanical Characteristics on Body Segments of Koreans", Applied Human Science, 18(3), 91-99, 1999.

Sederberg, T. W. and Parry, S. R., "Free-form deformation of solid geometric models", ACM Siggraph Computer, 20(4), 18-22, 1986.
Size Korea Home page, http://sizekorea.kats.go.kr(retrieved June 24, 2011). Sivapalan, S., et al. "3D Ellipsoid Fitting for Multi-view Gait Recognition", 8th IEEE International Conference on Advanced Video and SignalBased Surveillance, 355-360, 2011.

Solina, F. and Bajcsy, R., "Recovery of Parametrci Models from Range Images: The Case for Superquadrics with Global Deformations", IEEE Transactions on pattern analysis and machine intellicence, 12(2), 131-147, 1990.

William, H. P., et al. Numerical Recipes in $C, 2^{\text {nd }}$ ed., Cambridge University Press, 1992.

William, M. H., et al. "Direct Manipulation of Free-Form Deformations", Computer Graphics, 26(2), 177-184, 1992.

Xiao, Y. and Paul, J., "Building Superquadric Men from 3D whole-Body scan data", 4th IEEE Chapter Conference on Applied Cybernetics, 82-88, 2005. 
Appendix 1. Expressions for Joint Center Locations for Human Adults(GEBOD manual, 1994)

\begin{tabular}{l|c|c|c}
\hline JOINT & $\mathrm{X}$ & $\mathrm{Y}$ & $\mathrm{Z}$ \\
\hline Head-Neck & $\left(\mathrm{L}_{1 \mathrm{X}}+\mathrm{L}_{2 \mathrm{X}}\right) / 2$ & $\left(\mathrm{~L}_{1 \mathrm{Y}}+\mathrm{L}_{2 \mathrm{Y}}\right) / 2$ & $\left(\mathrm{~L}_{1 \mathrm{Z}}+\mathrm{L}_{2 \mathrm{Z}}\right) / 2-1.1811$ \\
\hline Neck-Thorax & $\mathrm{L}_{3 \mathrm{X}}+2.0079$ & $\mathrm{~L}_{3 \mathrm{Y}}$ & $\mathrm{L}_{3 \mathrm{Z}}-0.9843$ \\
\hline Thorax-Abdomen & $\mathrm{L}_{4 \mathrm{X}}+2.0079$ & $\mathrm{~L}_{4 \mathrm{Y}}$ & $\mathrm{L}_{4 \mathrm{Z}}$ \\
\hline Abdomen-Pelvis & $\mathrm{L}_{5 \mathrm{X}}+2.0079$ & $\mathrm{~L}_{5 \mathrm{Y}}$ & $\mathrm{L}_{6 \mathrm{Z}}-1.4961$ \\
\hline Right Shoulder & $\mathrm{L}_{6 \mathrm{X}}$ & $\mathrm{L}_{6 \mathrm{Y}}+1.4961$ & $\left(\mathrm{~L}_{7 \mathrm{Z}}+\mathrm{L}_{8 \mathrm{Z}}\right) / 2$ \\
\hline Right Elbow & $\left(\mathrm{L}_{7 \mathrm{X}}+\mathrm{L}_{8 \mathrm{X}}\right) / 2$ & $\left(\mathrm{~L}_{7 \mathrm{Y}}+\mathrm{L}_{8 \mathrm{Y}}\right) / 2$ & $\left(\mathrm{~L}_{9 \mathrm{Z}}+\mathrm{L}_{10 \mathrm{Z}}\right) / 2$ \\
\hline Right Wrist & $\left(\mathrm{L}_{9 \mathrm{X}}+\mathrm{L}_{10 \mathrm{X}}\right) / 2$ & $\left(\mathrm{~L}_{9 \mathrm{Y}}+\mathrm{L}_{10 \mathrm{Y}}\right) / 2$ & $\left(\mathrm{~L}_{112 \mathrm{Z}}-1.4961\right.$ \\
\hline Left Shoulder & $\mathrm{L}_{11 \mathrm{X}}$ & $\mathrm{L}_{11 \mathrm{Y}}-1.4961$ & $\left(\mathrm{~L}_{14 \mathrm{Z}}+\mathrm{L}_{15 \mathrm{Z}}\right) / 2$ \\
\hline Left Elbow & $\left(\mathrm{L}_{12 \mathrm{X}}+\mathrm{L}_{13 \mathrm{X}}\right) / 2$ & $\left(\mathrm{~L}_{12 \mathrm{Y}}+\mathrm{L}_{13 \mathrm{Y}}\right) / 2$ & $\left(\mathrm{~L}_{17 \mathrm{Z}}+\mathrm{L}_{18 \mathrm{Z}}\right) / 2-0.5906$ \\
\hline Left Wrist & $\left(\mathrm{L}_{14 \mathrm{X}}+\mathrm{L}_{15 \mathrm{X}}\right) / 2$ & $\left(\mathrm{~L}_{17 \mathrm{Y}}+\mathrm{L}_{18 \mathrm{Y}}\right) / 2$ & $\left(\mathrm{~L}_{19 \mathrm{Z}}+\mathrm{L}_{20 \mathrm{Z}}\right) / 2$ \\
\hline Right Hip & $\mathrm{L}_{16 \mathrm{X}}$ & $\left(\mathrm{L}_{19 \mathrm{Y}}+\mathrm{L}_{20 \mathrm{Y}}\right) / 2$ & $\left(\mathrm{~L}_{21 \mathrm{Z}}+\mathrm{L}_{22 \mathrm{Z}}\right) / 2$ \\
\hline Right Knee & $\left(\mathrm{L}_{19 \mathrm{X}}+\mathrm{L}_{20 \mathrm{X}}\right) / 2$ & $\left(\mathrm{~L}_{21 \mathrm{Y}}+\mathrm{L}_{22 \mathrm{Y}}\right) / 2$ & $\left(\mathrm{~L}_{18 \mathrm{Z}}+\mathrm{L}_{24 \mathrm{Z}}\right) / 2-0.5906$ \\
\hline Right Ankle & $\left(\mathrm{L}_{21 \mathrm{X}}+\mathrm{L}_{22 \mathrm{X}}\right) / 2$ & $\left(\mathrm{~L}_{18 \mathrm{Y}}+\mathrm{L}_{24 \mathrm{Y}}\right) / 2$ & $\left(\mathrm{~L}_{25 \mathrm{Z}}+\mathrm{L}_{26 \mathrm{Z}}\right) / 2$ \\
\hline Left Hip & $\mathrm{L}_{23 \mathrm{X}}$ & $\left(\mathrm{L}_{25 \mathrm{Y}}+\mathrm{L}_{26 \mathrm{Y}}\right) / 2$ & $\left(\mathrm{~L}_{27 \mathrm{Z}}+\mathrm{L}_{28 \mathrm{Z}}\right) / 2$ \\
\hline Left Knee & $\left(\mathrm{L}_{25 \mathrm{X}}+\mathrm{L}_{26 \mathrm{X}}\right) / 2$ & $\left(\mathrm{~L}_{27 \mathrm{Y}}+\mathrm{L}_{28 \mathrm{Y}}\right) / 2$ &
\end{tabular}

Appendix 2. Landmark Name(GEBOD manual, 1994)

\begin{tabular}{c|c|c|c}
\hline Landmark & Landmark Name & Landmark & Landmark Name \\
\hline $\mathrm{L}_{1}$ & Left Tragion & $\mathrm{L}_{15}$ & Left Ulnar Styloid \\
\hline $\mathrm{L}_{2}$ & Right Tragion & $\mathrm{L}_{16}$ & Right Trochanterion \\
\hline $\mathrm{L}_{3}$ & Cervicale & $\mathrm{L}_{17}$ & Right Anterior Superior Iliac Spline \\
\hline $\mathrm{L}_{4}$ & Tenth Rib Midspine & $\mathrm{L}_{18}$ & Symphysion \\
\hline $\mathrm{L}_{5}$ & Posterior Superior Iliac Midspine & $\mathrm{L}_{19}$ & Right Lateral Femoral Epicondyle \\
\hline $\mathrm{L}_{6}$ & Right Acromion & $\mathrm{L}_{20}$ & Right Medial Femoral Epicondyle \\
\hline $\mathrm{L}_{7}$ & Right Medial Humeral Epicondyle & $\mathrm{L}_{21}$ & Right Medial Malleolus \\
\hline $\mathrm{L}_{8}$ & Right Lateral Humeral Epicondyle & $\mathrm{L}_{22}$ & Right Lateral Malleolus \\
\hline $\mathrm{L}_{9}$ & Right Radial Styloid & $\mathrm{L}_{23}$ & Left Trochanterion \\
\hline $\mathrm{L}_{10}$ & Right Ulnar Styloid & $\mathrm{L}_{24}$ & Left Anterior Superior Iliac Spine \\
\hline $\mathrm{L}_{11}$ & Left Acromion & $\mathrm{L}_{25}$ & Left Lateral Femoral Epicondyle \\
\hline $\mathrm{L}_{12}$ & Left Medial Humeral Epicondyle & $\mathrm{L}_{26}$ & Left Medial Femoral Epicondyle \\
\hline $\mathrm{L}_{13}$ & Left Lateral Humeral Epicondyle & $\mathrm{L}_{27}$ & Left Medial Malleolus \\
\hline $\mathrm{L}_{14}$ & Left Radial Styloid & $\mathrm{L}_{28}$ & Left Lateral Malleolus \\
\hline
\end{tabular}




\section{Author listings}

Hyun Min Lee: last-nation@hanmail.net

Highest degree: BE, Department of Mechanical Design Engineering, Chonbuk National University

Position title: Master course, Department of Mechanical Engineering, Hanyang University

Areas of interest: Bio-CAD, Human Modeling, Dynamic Analysis, Numerical Optimization Techniques

Jay Jung Kim: jaykim@hanyang.ac.kr

Highest degree: $\mathrm{PhD}$, Department of Mechanical Engineering, Massachusetts Institute of Technology

Position title: Professor, Department of Mechanical Engineering, Hanyang University

Areas of interest: Geometric Modeling, CAD/CAM application, PDM/PLM, Rapid Prototyping, CATIA application

\section{Je Wook Chae: cjw@add.re.kr}

Highest degree: Ph.D department of mechanical engineering, Chungnam National University

Position title: Principal Researcher, Agency for Defense Development Areas of interest: Human modeling \& simulation, Biomechanics, Future Soldier

Date Received : 2011-07-25

Date Revised :2011-11-17

Date Accepted : 2011-11-17 